\title{
Outpatient surgery in patients with ankle fractures minimises hospital admissions and utilisation of healthcare resources
}

\author{
Travis S. Bullock ${ }^{1} \cdot$ Jose M. Gutierrez-Naranjo ${ }^{2} \cdot$ Robert G. DelBello $^{1} \cdot$ Ravi A. Karia ${ }^{2} \cdot$ Boris A. Zelle ${ }^{2}$
}

Received: 16 July 2020 / Accepted: 30 July 2020 / Published online: 8 August 2020

(C) SICOT aisbl 2020

\begin{abstract}
Purpose The recent outbreak of the novel coronavirus (SARS-CoV-2) has emphasised the need to minimise hospital admissions and utilisation of healthcare resources. The purpose of this study was to examine the outcomes of an outpatient surgery protocol for acute closed ankle fractures.

Methods In this retrospective study, 262 patients underwent outpatient surgery for their closed ankle fractures at our level-1 trauma centre. A total of 196 patients met our inclusion criteria and were ultimately included in the final analysis. Our primary outcomes' measures included post-operative admission to the emergency department within 30 days after surgery and unplanned hospital readmission within 30 days after surgery. Our secondary outcome measure included the incidence of surgical site infection (SSI) within 12 weeks after surgery.

Results Thirty-two patients $(16.3 \%$ ) had an unplanned emergency department visit within 30 days of fracture fixation and two patients $(1.0 \%)$ required hospital readmission within 30 days of their surgery. Sixteen patients $(8.2 \%)$ developed SSI, which included $11(5.6 \%)$ superficial and five (2.6\%) deep infections.

Conclusion Strategic outpatient management of acute closed ankle fractures is associated with acceptable rates of unplanned emergency department visits, hospital readmissions, and SSIs. In the context of the recent SARS-CoV-2 outbreak, outpatient management of these injuries may aide in the mitigation of nosocomial infections and the preservation of finite healthcare resources.
\end{abstract}

Keywords Ankle fracture $\cdot$ COVID-19 $\cdot$ SARS-CoV-2 $\cdot$ Outpatient $\cdot$ Open reduction and internal fixation

\section{Introduction}

Since its emergence, the novel coronavirus (SARS-CoV-2) and its subsequently named disease (COVID-19) has raised concerns regarding the availability of hospital beds and healthcare resources throughout the international community. As of June 14, 2020, the World Health Organization has reported over seven million confirmed cases and over four hundred thousand deaths in 216 countries [1]. In response, most healthcare systems have prioritised the conservation of disposable and non-disposable resources, with further efforts aimed

Boris A. Zelle

zelle@uthscsa.edu

1 Long School of Medicine, UT Health San Antonio, San Antonio, TX 78229-3900, USA

2 Department of Orthopaedics, UT Health San Antonio, Floyd Curl Dr, MC 7774, San Antonio, TX 78229-3900, USA at maintaining a low hospital census. This has largely been borne out of a necessity to free up resources for potential surges in COVID-19 admissions and the goal of minimising nosocomial viral transmission.

The COVID-19 outbreak has also had a significant impact on the orthopaedic community. Many local and state governments placed a moratorium on elective surgical procedures, thereby limiting surgery to patients that were deemed to have a serious medical illness, needed surgery to preserve life or $\operatorname{limb}(\mathrm{s})$, and those who without timely intervention would suffer serious adverse medical consequences [2]. Regarding orthopaedic trauma care, surgical fracture treatment is generally considered an essential surgical intervention under these criteria [3]. Thus, ethical considerations demand that patients with acute fractures continue to require hospital admission and timely surgical management. Yet, orthopaedic surgeons must also grapple with the socioeconomic impact of their decisions and continue to explore opportunities for minimising hospital admission and utilisation of healthcare resources [4]. While in 
most situations, traumatic fracture care inevitably requires inpatient management, there are subsets of patients who may be treated through an outpatient approach. Specifically, blanket inpatient admissions for traumatic closed ankle fractures has recently been called into question, with some studies showing increased cost-savings and improved outcomes with targeted outpatient treatment [5-9]. In the context of the recent COVID-19 outbreak, outpatient surgery for ankle fractures is a potentially valuable tool, as it may minimise the risk of nosocomial spread to other vulnerable patients or frontline healthcare workers [10-12]. Moreover, the prevalence of COVID-19 in the orthopaedic trauma population may be nine times higher than that of the general population, according to a recent study by Hernigou et al. [13]. Outpatient surgery also has the potential to control allocation of finite resources, such as personal protective equipment (PPE), hospital beds, and human resources [5-9, 14].

Although the SARS-CoV-2 outbreak has led to the publication of numerous related orthopaedic manuscripts, few studies to date have provided guidance for outpatient surgical treatment of traumatic fractures. The purpose of this study was to share our years-long experience with outpatient surgical fixation of traumatic closed ankle fractures, and to report on the outcomes for these patients. We hypothesize that outpatient surgery for ankle fractures is associated with low rates of readmission and post-operative complications.

\section{Materials and methods}

This study was approved by the institutional review board (IRB) of our institution. We performed a retrospective review of all consecutive patients with closed traumatic ankle fractures who underwent open reduction and internal fixation at our university-based accredited level-1 trauma centre between January 2014 and September 2016. We identified patients using our coding database and the Current Procedural Terminology (CPT) codes, 27766, 27769, 27792, 27814, 27822,27823 , and 27829. Patients with open ankle fractures, or age less than 18 years at the time of injury, were excluded. Routine follow-up appointments were advised at two weeks, six weeks, 12 weeks, and six months post-operatively. Patients who did not complete their 12-week follow-up appointment were excluded from this study, unless they had a previously documented SSI, ED visit, or readmission. Patients who were admitted to the hospital and treated as inpatients were excluded. Demographic and clinical data were extracted from the electronic medical records (EMR). The overall health status was categorized according to the American Society of Anaesthesiologists (ASA) score [15]. All fractures were classified based on preoperative standard radiographs according to the OTA/AO classification system [16].
The primary outcomes' measures included post-operative admission to the emergency department (ED) within 30 days of surgery and unplanned hospital readmission within 30 days of surgery. All ED admissions and hospital readmissions were recorded from the EMR along with the admission diagnoses. Our secondary outcome measure included the incidence of SSI, which were defined as either superficial or deep according to the Centers for Disease Control and Prevention (CDC) criteria [17].

\section{Peri-operative protocol}

All patients in this study were initially evaluated in the ED at which time the diagnosis was established and appropriate management was initiated including closed fracture manipulation if necessary, splinting, and pharmacological pain control. Patients were assessed for feasibility of outpatient surgery based on patient and surgeon preference, operating room availability, medical co-morbidities, and social circumstances. Pre-operative evaluation including lab work, electrocardiogram, and chest radiograph was obtained as necessary. Applicable forms including surgical consent and surgery scheduling cards were then completed in the ED. Prior to being discharged, patients were provided further education on warning signs necessitating medical attention, non-weight bearing instructions, lower extremity elevation, and at home injury care. In addition, patients received standard pre-operative instructions as per institutional protocol. Patients with significant soft tissue swelling were scheduled for pre-operative skin checks in our outpatient clinic. On the day before surgery, patients were contacted via telephone to confirm the time of surgery and counselled on pre-operative instructions, in particular to remain fasted (NPO) after midnight.

On the day of surgery, patients were offered peripheral nerve blocks by the anaesthesia team. Prior to surgery, all patients received pre-operative intravenous (IV) antibiotics according to standard hospital protocol. Post-operatively, additional doses of IV antibiotics were given within the standard dosing intervals while patients awaited discharge from the same day surgery unit. Of note, discharges were not delayed for the purpose of providing additional doses of antibiotics. Post-operatively, patients received serial neurovascular checks and appropriate pain management. Patients were then discharged after being deemed medically stable and fulfilling discharge criteria as per institutional protocol. Patients were provided standard discharge instructions and scheduled for a follow-up appointment two weeks after surgery.

\section{Results}

\section{Patient demographics}

A total of 545 patients with closed ankle fractures were treated with open reduction and internal fixation at our institution 
during the study period, whereby a total of 262 patients underwent outpatient surgery. Following application of our inclusion and exclusion criteria, 196 patients were included in the final analysis with a mean follow-up of 28.1 weeks (median: 21.8). Patient demographics and clinical data are further detailed in Table 1. In our study cohort, the mean age was $38.9 \pm 14.9$ years (range 18-95) and $50.5 \%$ of patients were female. Of note, $48.5 \%$ of patients enrolled in this study were considered obese (body mass index $>30.0 \mathrm{~kg} / \mathrm{m}^{2}$ ), $31.1 \%$ disclosed that they were tobacco users, and approximately $13.8 \%$ of patients had pre-existing diabetes mellitus. Most patients sustained their ankle fracture through a same level injury, such as twisting and ground-level falls ( $n=134$; $68.4 \%)$. The majority of fractures were classified as OTA/AO type $44 \mathrm{~B}(n=142 ; 72.4 \%)$. The overall health status was classified as ASA 2 in 120 patients (61.2\%). Most patients included in this study received a peripheral nerve block (167 patients, $85.2 \%$ ).

\section{Post-operative outcomes}

In terms of our primary outcome measures, 32 patients (16.3\%) required a total of 35 unplanned $E D$ visits within 30 days of their surgery. Of these 35 visits, the most commonly recorded chief complaint was pain associated with the prior orthopaedic injury $(n=19 ; 54.3 \%)$. The next most common was a medical complaint unrelated to their ankle injury $(n=6 ; 17.1 \%)$ (Table 2).

Additionally, two patients required three readmissions within 30 days of their surgery. One patient was readmitted for a pulmonary embolus on post-operative day 14 . The patient was successfully treated for his pulmonary embolus and was discharged five days later. Unfortunately, this patient required a second hospital readmission on post-operative day 21 for sepsis secondary to an epidural abscess. The patient again received appropriate medical management and was discharged 12 days later without further incident. Another patient was readmitted 20 days postoperatively for a superficial SSI. This patient received intravenous (IV) antibiotics alone and was discharged three days later on oral antibiotics requiring no further interventions for infection.

In respect to our secondary outcomes measures, 16 patients $(8.2 \%)$ developed SSIs. Of these 16 infections, 11 (5.6\%) were considered superficial infections and five $(2.6 \%)$ were considered deep. Of the 11 patients with superficial infections, ten patients were successfully treated with oral antibiotics and/or local wound care alone. One other patient (as mentioned above) required three days of IV antibiotics and healed without further intervention. In regard to patients with deep infections, all patients received surgical irrigation and debridement (I\&D), implant removal, and/or extended antibiotic treatment. Of note, only one of these patients was readmitted within 30 days of their surgery for a deep infection, while the other four deep infections required readmission beyond the 30-day time point.
Table 1 Demographic and clinical data

\begin{tabular}{|c|c|}
\hline \multicolumn{2}{|l|}{ Age, years } \\
\hline Mean \pm SD & $38.9 \pm 14.9$ \\
\hline Median (min, max) & $36(18,95)$ \\
\hline Gender & $N(\%)$ \\
\hline Male & $97(49.5)$ \\
\hline Female & $99(50.5)$ \\
\hline \multicolumn{2}{|l|}{ BMI, $\mathrm{kg} / \mathrm{m}^{2}$} \\
\hline Normal (BMI: 18.5-24.9) & $36(18.4)$ \\
\hline Overweight (BMI:25-29.9) & $65(33.2)$ \\
\hline Obese (BMI: > 30.0) & $95(48.5)$ \\
\hline \multicolumn{2}{|l|}{ Co-morbidities/medical history } \\
\hline Alcohol use & $108(55.1)$ \\
\hline Tobacco use & $61(31.1)$ \\
\hline Illicit drug use & $44(22.4)$ \\
\hline Immune compromise & $3(1.5)$ \\
\hline Diabetes mellitus & $27(13.8)$ \\
\hline \multicolumn{2}{|l|}{ ASA class ${ }^{\#}$} \\
\hline 1 & $25(12.8)$ \\
\hline 2 & $120(61.2)$ \\
\hline 3 & $51(26.0)$ \\
\hline \multicolumn{2}{|l|}{ Mechanism of injury } \\
\hline Motor vehicle collision & $1(0.5)$ \\
\hline Motorcycle collision & $5(2.6)$ \\
\hline Fall from height & $44(22.4)$ \\
\hline Same level injury & $134(68.4)$ \\
\hline Crush injury & $9(4.6)$ \\
\hline Motor vehicle vs. pedestrian & $1(0.5)$ \\
\hline N/A & $2(1.0)$ \\
\hline \multicolumn{2}{|l|}{ OTA classification } \\
\hline $44 \mathrm{~A}$ & $6(3.1)$ \\
\hline 44B & $142(72.4)$ \\
\hline $44 \mathrm{C}$ & $41(20.9)$ \\
\hline 43B1 & $7(3.6)$ \\
\hline \multicolumn{2}{|l|}{ Ankle fracture with dislocation } \\
\hline Yes & $26(13.3)$ \\
\hline No & $170(86.7)$ \\
\hline \multicolumn{2}{|l|}{ Received a peripheral nerve block } \\
\hline Yes & $167(85.2)$ \\
\hline No & $29(14.8)$ \\
\hline
\end{tabular}

\#American Society of Anaesthesiologists physical status classification

\section{Discussion}

It remains the obligation of the orthopaedic community to minimise utilisation of healthcare resources when making treatment decisions and establishing treatment protocols. The current state of the SARS-CoV-2 outbreak has further emphasised this issue. A primary strategic goal of many healthcare systems during the COVID-19 outbreak has been 
Table 2 Unplanned ED visits, readmissions, and surgical site infections

\begin{tabular}{ll}
\hline & $N(\%)$ \\
\hline Patients requiring unplanned ED visits $^{*}$ & $32(16.3)$ \\
Frequency of ED visits by chief complaint ${ }^{*}(N=35)$ & \\
Pain related to orthopaedic injury & $19(54.3)$ \\
Medical complaint (non-orthopaedic) & $6(17.1)$ \\
New injury & $3(8.6)$ \\
Concern for SSI infection & $3(8.6)$ \\
Shortness of breath or concern for pulmonary embolus & $2(5.7)$ \\
Bandage, cast, or splint issues & $1(2.9)$ \\
Concern for sepsis & $1(2.9)$ \\
Patients requiring readmission* & $2(1.0)$ \\
Frequency of readmissions by admitting diagnosis* $(N=3)$ & \\
Pulmonary embolus & $1(33.3)$ \\
Superficial surgical site infection & $1(33.3)$ \\
Sepsis & $1(33.3)$ \\
Surgical site infections $(N=16)$ & \\
Superficial infection & $11(5.6)$ \\
Deep infection & $5(2.6)$ \\
\hline
\end{tabular}

*Emergency department visits and/or readmissions occurring within 30 days of operative treatment

to minimise utilisation of healthcare resources and the number of hospital admissions in preparation for an influx of COVID19 patients. The orthopaedic community was largely affected by these efforts as the suspension of elective surgical procedures played a key factor in achieving this objective $[2,3]$. Yet, acute orthopaedic trauma care has overall continued to require hospital admissions and subsequent surgical treatments as ethical considerations demand appropriate and timely treatment in order to avoid adverse long-term consequences. However, there remain opportunities for orthopaedic trauma surgeons to contribute to the public health effort of resource preservation. For instance, select patients with isolated ankle fractures may be amenable to discharge from the ED after their initial evaluation, with subsequent outpatient surgery and no hospital admission [5-9]. Our study data shows that outpatient surgical fixation represents a reasonable approach to these injuries, as we demonstrated acceptable rates of unplanned ED visits, hospital readmissions, and SSIs. Based on these findings, we suggest that a safe and properly implemented outpatient protocol may be beneficial in mitigating the risk of inpatient viral transmission, safeguarding frontline healthcare workers, and conserving finite resources.

Examining unplanned returns to care after surgery has largely been employed as a quality improvement metric [18], but now has garnered importance from a public health perspective, given the current pandemic. Two previous studies examining the rate of 30-day unplanned ED visits after foot and ankle surgery report rates of $11-41 \%[18,19]$. However, when one study isolated cases to outpatient surgery and surgery-related complaints, this range narrowed to approximately $11-17 \%$, with pain being amongst the most common reasons for ED presentation [19, 20]. In terms of readmissions, two larger studies investigating readmissions after surgical fixation of an ankle fracture showed readmission rates varying from approximately $3-5 \%$, with infections being reported as the most common cause in one study [21, 22]. Interestingly, these results are similar with the elective orthopaedic surgery data. Recently, a national database study of 44,120 patients undergoing an elective orthopaedic surgery showed that $3.8 \%$ of patients had an all cause readmission, with surgical site complications accounting for most cases [23]. Furthermore, two studies that utilised generally accepted exclusions for elective outpatient total joint arthroplasty (e.g. renal failure, liver cirrhosis, cardiovascular disease, pulmonary disease, poor social support) found no significant difference in terms of readmissions or unplanned ED visits when compared with patients treated as an inpatient $[24,25]$. Our study aligns with these previous reports showing comparable rates of readmissions and unplanned ED visits, with pain and infections being the most common culprits. However, the previous literature and our results underscore areas of needed improvement. For instance, patients suffering from post-operative pain or those with surgical site concerns may be advised through virtual orthopaedic assessments or telemedicine, which has recently been described as a useful tool in the context of the COVID-19 outbreak [26].

Considering that SSI are a common cause of readmissions and unplanned ED visits, confronting this challenge is crucial. The reported incidence of SSI after open reduction and internal fixation of ankle fractures varies from approximately 2.6$19 \%$ for superficial infections and $1.1-10 \%$ for deep infections [27-34]. However, many of these studies included both open and closed injuries, had varying criteria for SSI, and were mostly based on inpatient surgery [34]. Thus, the infection rates observed in this study compare favourably with reports from the literature. In this context, we would also like to emphasise the potential benefit of outpatient surgery for controlling nosocomial infections as it has been suggested that longer pre-operative admissions for surgical fixation of ankle fractures may increase rates, thereby increasing downstream resource utilisation and hospital staffing demands [5, 27]. Thus, we feel that outpatient surgery in ankle fractures may be associated with satisfactory low rates of SSIs, while also having the added benefits of social distancing, shorter preoperative hospital exposure, and decreased healthcare utilisation [5-9].

Our study is not without limitations. The study was conducted retrospectively which inherently introduces the risk of bias. Moreover, most data points were extracted from the electronic medical records, which present the opportunity for errors in the data retrieval and recording process. Furthermore, 
all ED visits and readmission data are limited in that only events occurring at our institution were recorded, leaving the possibility that some visits or readmissions occurring at outside healthcare networks went undocumented. Finally, our study reports on a selective patient population. Thus, only patients that were deemed appropriate for outpatient surgery were enrolled in this protocol. The decision as to whether patients during the study period were treated as inpatients versus outpatients was based on several factors, such as patient and surgeon preference, operating room availability, medical co-morbidities, and social circumstances. Therefore, future studies will have to identify specific criteria that will allow for identifying which specific subset of patients is amenable to outpatient ankle fracture surgery.

\section{Conclusion}

In conclusion, our study results and comparisons with the recent literature indicate that outpatient surgery is a safe and reasonable approach for a selected group of patients with closed ankle fractures. Our study demonstrated acceptable results in terms of 30-day unplanned ED visits, 30-day readmissions, and SSIs. In particular, during the current circumstances of the COVID-19 outbreak, outpatient fracture fixation should be considered when feasible to help minimise the utilisation of valuable healthcare resources and prevent further nosocomial viral transmission.

\section{References}

1. World Health Organization (2020) Coronavirus disease (COVID19) outbreak. https://www.who.int/emergencies/diseases/novelcoronavirus-2019. Accessed 14 June 2020

2. American College of Surgeons (2020) COVID-19: executive orders by state on dental, medical, and surgical procedures. https://www. facs.org/covid-19/legislative-regulatory/executive-orders. Accessed 14 June 2020

3. Dephillipo NN, Larson CM, O'neill OR, Laprade RF (2020) Guidelines for ambulatory surgery centers for the care of surgically necessary/time-sensitive orthopaedic cases during the COVID-19 pandemic. J Bone Joint Surg Am 102:933-936

4. Randall RM, Rafferty R (2020) Philosophy problems become real during the COVID-19 pandemic. J Bone Joint Surg Am. https://doi. org/10.2106/JBJS.20.00512

5. Stull JD, Bhat SB, Kane JM, Raikin SM (2017) Economic burden of inpatient admission of ankle fractures. Foot Ankle Int 38:9971004

6. Bhandari M, Sprague S, Ayeni OR, Hanson BP, Moro JK (2004) A prospective cost analysis following operative treatment of unstable ankle fractures: 30 patients followed for 1 year. Acta Orthop Scand 75:100-105

7. Lloyd JM, Martin R, Rajagopolan S, Zieneh N, Hartley R (2010) An innovative and cost-effective way of managing ankle fractures prior to surgery — home therapy. Ann R Coll Surg Engl 92:615-618
8. Qin C, Dekker R, Blough J, Kadakia A (2016) Safety and outcomes of inpatient compared with outpatient surgical procedures for ankle fractures. J Bone Joint Surg Am 98:1699-1705

9. Khakha R, Berber O, Patel A, Kurar L, James L (2020) Ankle home stay programme: a review of ankle fracture management and costs at a busy district general hospital. Ann Med Surg (Lond) 50:6-9

10. Wee LE, Conceicao EP, Sim XYJ et al (2020) Minimizing intrahospital transmission of COVID-19: the role of social distancing. J Hosp Infect 105:113-115

11. Guan WJ, Ni ZY, Hu Y et al (2020) Clinical characteristics of coronavirus disease 2019 in China. N Engl J Med 382:1708-1720

12. Bai $Y$, Yao L, Wei $T$ et al (2020) Presumed asymptomatic carrier transmission of COVID-19. JAMA 323:1406-1407

13. Hernigou J, Morel X, Callewier A, Bath O, Hernigou P (2020) Staying home during "COVID-19" decreased fractures, but trauma did not quarantine in one hundred and twelve adults and twentyeight children and the "tsunami of recommendations" could not lockdown twelve elective operations. Int Orthop. https://doi.org/ 10.1007/s00264-020-04619-5

14. Emanuel EJ, Persad G, Upshur R et al (2020) Fair allocation of scarce medical resources in the time of COVID-19. N Engl J Med 382:2049-2055

15. American Society of Anesthesiologists (1963) New classification of physical status. Anesthesiology 24:111

16. Meinberg EG, Agel J, Roberts CS et al (2018) Fracture and dislocation classification compendium. J Orthop Trauma 32:S1-S170

17. Centers for Disease Control and Prevention/ National Healthcare Safety Network (2020) Surgical site infection. https://www.cdc. gov/nhsn/pdfs/pscmanual/pcsmanual_current. Accessed 14 June 2020

18. Shibuya N, Patel H, Graney C, Jupiter DC (2018) Factors associated with emergency room visits within 30 days of outpatient foot and ankle surgeries. Proc (Baylor Univ Med Cent) 31:157-160

19. Abousayed MM, Johnson CK, Moral M, Sternbach S, Rosenbaum AJ (2019) Trends in urgent care utilization following ankle fracture fixation. Foot Ankle Int 40:218-223

20. Shibuya N, Graney C, Patel H, Jupiter DC (2018) Predictors for surgery-related emergency department visits within 30 days of foot and ankle surgeries. J Foot Ankle Surg 57:1101-1104

21. Basques BA, Miller CP, Golinvaux NS, Bohl DD, Grauer JN (2015) Morbidity and readmission after open reduction and internal fixation of ankle fractures are associated with preoperative patient characteristics. Clin Orthop Relat Res 473:1133-1139

22. Varacallo MA, Mattern P, Acosta J, Toossi N, Denehy KM, Harding SP (2018) Cost determinants in the 90-day management of isolated ankle fractures at a large urban academic hospital. J Orthop Trauma 32:338-343

23. Minhas SV, Kester BS, Lovecchio FC, Bosco JA (2017) Nationwide 30-day readmissions after elective orthopedic surgery: reasons and implications. J Healthc Qual 39:34-42

24. Gillis ME, Dobransky J, Dervin GF (2019) Defining growth potential and barriers to same day discharge total knee arthroplasty. Int Orthop 43:1387-1393

25. Paredes O, Ñunez R, Klaber I (2018) Successful initial experience with a novel outpatient total hip arthroplasty program in a public health system in Chile. Int Orthop 42:1783-1787

26. Tanaka MJ, Oh LS, Martin SD, Berkson EM (2020) Telemedicine in the era of COVID-19: the virtual orthopaedic examination. J Bone Joint Surg Am 102:pe57

27. Schepers T, De Vries MR, Van Lieshout EMM, Van der Elst M (2013) The timing of ankle fracture surgery and the effect on infectious complications: a case series and systematic review of the literature. Int Orthop 37:489-494

28. Thangarajah T, Prasad PS, Narayan B (2009) Surgical site infections following open reduction and internal fixation of ankle fractures. Open Orthop J 3:56-60 
29. Korim MT, Payne R, Bhatia M (2014) A case-control study of surgical site infection following operative fixation of fractures of the ankle in a large U.K. trauma unit. Bone Joint J 96-B:636-640

30. Ovaska MT, Mäkinen TJ, Madanat R et al (2013) Risk factors for deep surgical site infection following operative treatment of ankle fractures. J Bone Joint Surg Am 95:348-353

31. Miller AG, Margules A, Raikin SM (2012) Risk factors for wound complications after ankle fracture surgery. J Bone Joint Surg Am 94:2047-2052

32. Srinivasan CM, Moran CG (2001) Internal fixation of ankle fractures in the very elderly. Injury 32:559-563
33. Kelly EG, Cashman JP, Groarke PJ, Morris SF (2013) Risk factors for surgical site infection following operative ankle fracture fixation. Ir J Med Sci 182:453-456

34. Sun R, Li M, Wang X et al (2017) Surgical site infection following open reduction and internal fixation of a closed ankle fractures: a retrospective multicenter cohort study. Int J Surg 48:86-91

Publisher's note Springer Nature remains neutral with regard to jurisdictional claims in published maps and institutional affiliations. 